\title{
FACTORES DE RIESGO PARA EL NEONATO PEQUEÑO PARA LA EDAD GESTACIONAL EN UN HOSPITAL DE LIMA, PERÚ
}

\author{
J. Eduardo Tejeda-Mariaca1,a, Orion Pizango-Mallquii,a, Miguel Alburquerque-Duglio ${ }^{1, a}$, \\ Percy Mayta-Tristán ${ }^{1, b}$
}

\begin{abstract}
RESUMEN
Objetivos. Identificar factores de riesgo para neonatos a término pequeños para la edad gestacional. Materiales $y$ métodos. Cohorte retrospectiva que utilizó datos del Sistema Informático Materno Perinatal del Hospital María Auxiliadora de Lima, del período 2000 a 2010. Se evaluó la edad materna, paridad, nivel educativo, estado civil, índice de masa corporal pregestacional, número de controles prenatales, presencia de patologías como preeclampsia, eclampsia, infección urinaria y diabetes gestacional como factores de riesgo en pequeños para edad gestacional. El peso para la edad gestacional fue calculado sobre la base de percentiles peruanos. Se calcularon los riesgos relativos crudos (RR) y ajustados (RRa) con sus intervalos de confianza al 95\% usando modelos lineales generalizados log binomial. Resultados. Se incluyeron 64670 gestantes. La incidencia de pequeños para la edad gestacional fue 7,2\%. La preeclampsia (RRa 2,0; IC 95\%: 1,86-2,15), eclampsia (RRa 3,22; IC 95\%: 2,38-4,35), bajo peso materno (RRa 1,38; IC 95\%: 1,23-1,54), nuliparidad (RRa 1,32; IC 95\%: 1,23-1,42), edad $\geq 35$ años (RRa 1,16; IC 95\%: 1,04-1,29), tener controles prenatales de 0 a 2 (RRa 1,43; IC 95\%: 1,32-1,55) y 3 a 5 (RRa 1,22; IC 95\%: 1,14-1,32) fueron factores de riesgo para ser pequeños para la edad gestacional. Conclusiones. Es necesario identificar a las gestantes con factores de riesgo como los encontrados para disminuir la condición de pequeños para la edad gestacional. Se debe actuar poniendo énfasis en factores modificables, tales como la frecuencia de sus controles prenatales.
\end{abstract}

Palabras clave: Recién nacido pequeño para edad gestacional, Atención prenatal; Paridad; Embarazo; Índice de masa corporal (fuente: DeCS BIREME)

\section{RISK FACTORS OF SMALL FOR THE GESTATIONAL AGE NEONATES IN A HOSPITAL OF LIMA, PERU}

\begin{abstract}
Objectives. Identify risk factors for at-term small for gestational age newborns. Materials and methods. Retrospective cohort study using data from the Maternal Perinatal Information System of the Maria Auxiliadora Hospital of Lima, from the period 2000-2010. Maternal age, parity, education level, marital status, pregestational body mass index, number of prenatal care visits, presence of conditions such as preeclampsia, eclampsia, urinary tract infection and gestational diabetes as risk factors in small for gestational age newborns were evaluated. The weight for gestational age was calculated based on Peruvian percentiles. Crude relative risk (RR) and adjusted (ARR) were calculated with confidence intervals of $95 \%$ using log-binomial generalized linear models. Results. 64,670 pregnant women were included. The incidence for small for gestational age was $7.2 \%$. Preeclampsia (ARR $2.0,95 \% \mathrm{Cl}: 1.86$ to 2.15 ), eclampsia (ARR $3.22,95 \% \mathrm{Cl}: 2.38$ to 4.35 ), low maternal weight (ARR $1.38 ; 95 \% \mathrm{Cl}$ : 1.23 to 1.54 ), nulliparity (ARR $1.32,95 \% \mathrm{Cl}: 1.23$ to 1.42 ), age $\geq 35$ years (ARR $1.16,95 \% \mathrm{Cl}: 1.04-1.29$ ), having prenatal care visits from 0 to 2 (ARR $1.43,95 \% \mathrm{Cl}: 1.32$ to 1.55 ) and 3 to 5 (ARR $1.22,95 \% \mathrm{Cl}: 1.14$ to 1.32 ) were risk factors for small for gestational age. Conclusions. It is necessary to identify pregnant women with risk factors such as those found to decrease the condition of small for gestational age. Actions should emphasize modifiable factors, such as the frequency of prenatal care visits..
\end{abstract}

Key words: Infant, small for gestational age; Prenatal care; Parity; Pregnancy; Body mass index (source: MeSH NLM).

\footnotetext{
Escuela de Medicina, Universidad Peruana de Ciencias Aplicadas. Lima, Perú

Médico cirujano; ${ }^{\mathrm{b}}$ médico salubrista

Recibido: : 13-02-15 Aprobado: 13-05-15
}

Citar como: Tejeda-Mariaca JE, Pizango-Mallqui O, Alburquerque-Duglio M, Mayta-Tristán P. Factores de riesgo para el neonato pequeño para la edad gestacional en un hospital de Lima, Perú. Rev Peru Med Exp Salud Publica. 2015;32(3):449-56. 


\section{INTRODUCCIÓN}

La gestación implica una serie de cambios anatómicos y fisiológicos adaptativos necesarios para albergar a un nuevo organismo. Por lo tanto, la evaluación continua del estado de salud de la madre y el feto durante el embarazo resulta fundamental para evitar y manejar oportunamente las complicaciones. Asimismo, las características de la población materna a nivel mundial y regional exigen considerar factores de riesgo que pueden asociarse a resultados adversos en el producto de la gestación, así como identificar al paciente en riesgo de complicaciones. Este hecho constituye un reto en la salud materno-infantil (1).

Las complicaciones perinatales con mayor repercusión en morbilidad y mortalidad se encuentran con más frecuencia en grupos específicos como son los prematuros, recién nacidos de bajo peso y los pequeños para la edad gestacional. Dentro de estas complicaciones se encuentran las infecciones neonatales, el síndrome de dificultad respiratoria, los trastornos metabólicos, entre otras ${ }^{(2-4)}$. A partir de la evidencia señalada, es posible deducir que el estudio de los factores que predisponen a una mayor incidencia de estos grupos puede tener un impacto importante en la reducción de complicaciones perinatales ${ }^{(5)}$.

Relacionar el peso del recién nacido con la edad gestacional al nacer es una medida usada para determinar si el neonato se encuentra en un peso adecuado para la edad gestacional (AEG), al encontrarse entre el percentil 10 y 90; pequeño para la edad gestacional (PEG), si está por debajo del percentil 10; y grande para la edad gestacional (GEG), si se encuentra sobre el percentil 90 (6). Históricamente, la curva usada para esta clasificación ha sido la de Lubchenco, la cual se realizó en base a población de EE. UU. ${ }^{(6)}$. Sin embargo, la Organización Mundial de la Salud (OMS) recomienda utilizar curvas de referencia del peso de nacimiento para la edad gestacional elaboradas con población de la región. En el año 2007, Ticona et al. propusieron una curva de referencia peruana particularmente útil en estudios de investigación a nivel local, con el fin de evitar la subestimación del PEG en nuestra población. Esta fue desarrollada en base a una población de 50568 recién nacidos, de 29 hospitales de todo el Perú. La prevalencia de recién nacidos PEG fue mayor utilizando la nueva curva peruana en comparación con las curvas de Lubchenco, 10,1 y 4,1\% respectivamente ${ }^{(2)}$. El porcentaje de PEG de recién nacidos a término que se encontró en el estudio peruano fue mayor al de otros estudios en Latinoamérica (7).

Factores como el índice de masa corporal (IMC) pregestacional, el número de controles prenatales (CPN), el nivel socioeconómico, la paridad, la desnutrición, enfermedades como preeclampsia, entre otros, que guardan asociación con el recién nacido PEG. Sin embargo, estos resultados pueden variar según la población estudiada ${ }^{(8-13)}$. No se han encontrado trabajos que estudien los factores de riesgo asociados a los recién nacidos a término PEG utilizando la curva peruana. Asimismo, considerando la elevada incidencia del recién nacido PEG, resulta necesario conocer qué factores de riesgo en particular tienen mayor relevancia en la población peruana.

El objetivo del estudio es Identificar factores de riesgo para neonatos a término pequeños para edad en un hospital de Lima, Perú.

\section{MATERIALES Y MÉTODOS}

Se realizó un estudio de tipo cohorte retrospectiva basada en los datos registrados en el Sistema Informático Perinatal (SIP 2000) del Hospital María Auxiliadora (HMA) durante el período 2000-2010. Como centro de referencia en la atención materna en Lima Sur, Perú; este hospital está encargado de una población de 2 millones de habitantes y atiende en promedio 7000 partos por año. Los registros del SIP 2000 se obtienen a partir de los datos ingresados en la hoja CLAP (Centro Latinoamericano de Perinatología) de cada gestante ${ }^{(14)}$. Esta hoja contiene información de los antecedentes maternos, de la actual gestación y acerca de las características del parto y puerperio.

Se incluyó a aquellas mujeres con gestación única y a término (de 37 a 41 semanas de gestación) que iniciaron su CPN en los centros de salud de la jurisdicción del HMA o aquellas que iniciaron su CPN en el mismo hospital. Se excluyó mujeres con comorbilidades específicas como tuberculosis, infección por VIH, cáncer de cuello uterino, cáncer de mama, y a quienes presentaron datos incompletos (edad, peso pregestacional, talla, CPN, nivel educativo, paridad). Aquellas mujeres cuyos hijos no presentaron el dato de edad gestacional y peso, así como los que presentaron malformaciones congénitas (espina bífida, hidrocefalia congénita, otros defectos de sistema nervioso central, defectos cromosómicos, defectos congénitos múltiples) también fueron excluidas.

El peso para la edad gestacional se determinó a partir del peso del recién nacido y la edad gestacional, esta última calculada mediante la fecha de última regla o ecografía del primer trimestre registradas en SIP 2000. Se utilizó la curva peruana elaborada por Ticona et al. para el diagnóstico de PEG ${ }^{(2)}$. EI IMC pregestacional se calculó en base a la talla consignada y al peso habitual pregestacional, categorizándola en bajo peso $(<18,5)$, normopeso $(18,5-$ $24,9)$, sobrepeso $(25-29,9)$ y obesidad $(\geq 30)$. El número de CPN se dividió en rangos de 0-2, 3-5, 6-8 y $\geq 9$. 
Se incluyó otras variables importantes, tales como edad materna, estado civil, grado de instrucción, y número de partos, todas ellas obtenidas a partir del SIP 2000. Asimismo, se incluyó en el análisis enfermedades maternas, tales como preeclampsia, eclampsia, infección urinaria y diabetes gestacional.

La base de datos obtenida del SIP se exportó al programa Excell para Windows y luego fue procesada con el programa STATA v11.0. (StataCorp. TX, US). Las variables categóricas se describieron con frecuencias absolutas y relativas, mientras que las numéricas usando media y desviación estándar, así como mediana y rango intercuartílico. Se evaluó la asociación entre la condición de recién nacido PEG con las variables de interés usando la prueba de chi cuadrado. Se evaluó la magnitud de la asociación calculando los riesgos relativos crudos (RR) y ajustados (RRa) con sus respectivos intervalos de confianza al 95\% (IC 95\%) usando modelos lineales generalizados log binomial.
Las variables que resultaron significativas en el análisis bivariado fueron incluidas en el modelo ajustado. Se consideró un $p<0,05$ como significativo.

El protocolo de estudio fue revisado y aprobado por el Comité de Ética de la Universidad Peruana de Ciencias Aplicadas de Lima, Perú. Los datos se obtuvieron de una base anonimizada y no se trabajó directamente con las participantes. Por lo tanto, no se requirió de un consentimiento informado.

\section{RESULTADOS}

Se inició la selección con 78686 registros de gestantes. Luego de aplicar los criterios de exclusión y retirar los datos faltantes quedaron 64670 participantes. Se encontró una incidencia del 7,2\% para los recién nacidos PEG en esta muestra (Figura 1). En la Tabla 1 se muestra las características sociodemográficas

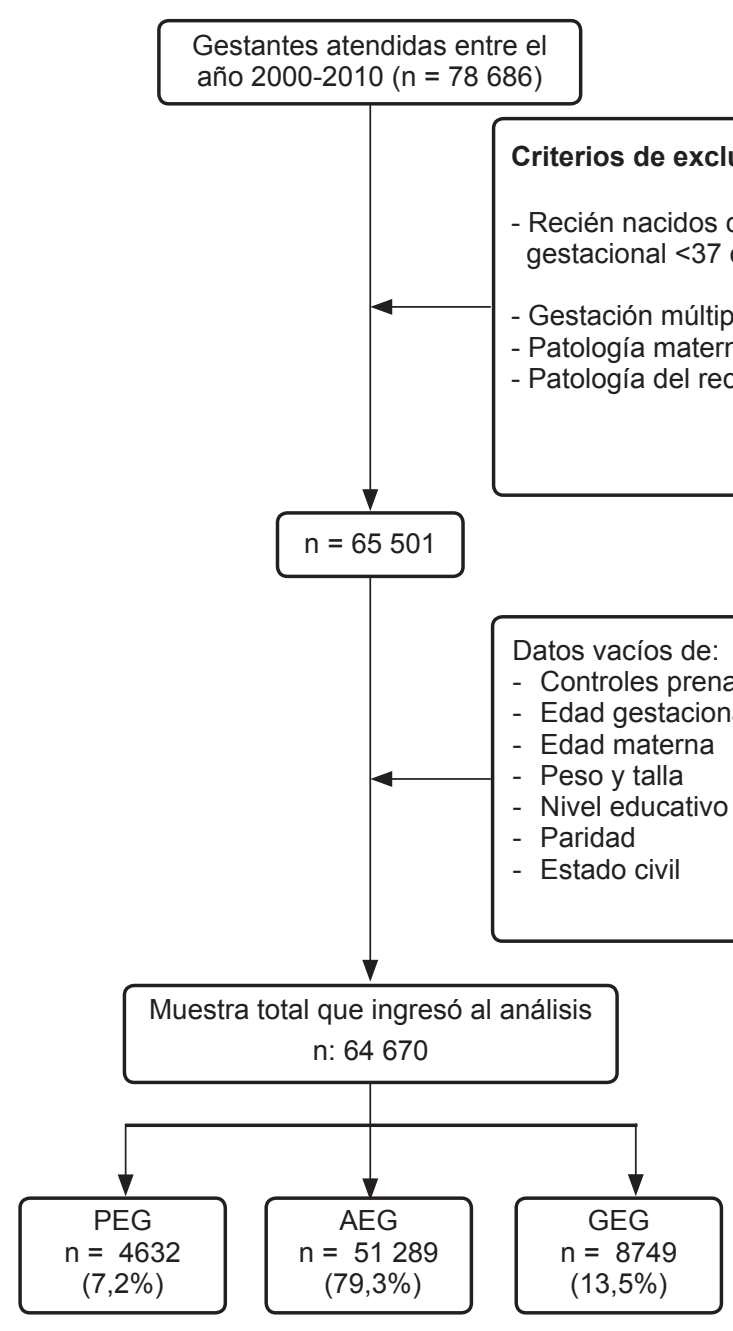

*Enfermedad materna específica: infección por VIH, infección por tuberculosis, cáncer de cérvix, cáncer de mama.

**Patología del recién nacido específica: defectos congénitos: espina bífida, hidrocefalia congénita, otros defectos de sistema nervioso central, defectos cromosómicos, defectos congénitos múltiples. PEG: pequeño para edad gestacional, AEG: adecuado para edad gestacional, GEG: grande para edad gestacional

Figura 1. Flujograma de selección: historias clínicas de gestantes atendidas en el Hospital María Auxiliadora entre el año 2000 y 2010 
Tabla 1. Características de las gestantes atendidas en el Hospital María Auxiliadora entre los años 2000 y 2010

\begin{tabular}{|c|c|c|}
\hline Variables & $n=64670$ & $(\%)$ \\
\hline Edad $^{*}$ & $25,7(6,7)$ & \\
\hline \multicolumn{3}{|l|}{ Edad } \\
\hline 20 a 35 años & 44709 & $(69,3)$ \\
\hline Menor de 20 años & 13347 & $(20,6)$ \\
\hline Mayor de 35 años & 6614 & $(10,2)$ \\
\hline \multicolumn{3}{|l|}{ Grado de instrucción } \\
\hline Superior & 7082 & $(10,9)$ \\
\hline Secundaria completa & 49422 & $(76,4)$ \\
\hline Primaria completa & 7707 & $(11,9)$ \\
\hline Ninguno & 459 & $(0,7)$ \\
\hline \multicolumn{3}{|l|}{ Estado civil } \\
\hline Casada o conviviente & 42319 & $(65,4)$ \\
\hline Soltera, divorciada o separada & 22351 & $(34,6)$ \\
\hline \multicolumn{3}{|l|}{ Paridad } \\
\hline 1-2 partos & 26374 & $(40,7)$ \\
\hline Ningún parto & 30733 & $(47,5)$ \\
\hline 3 a más partos & 7563 & $(11,6)$ \\
\hline \multicolumn{3}{|l|}{ Índice de masa corporal pregestacional } \\
\hline Normal & 41717 & $(64,5)$ \\
\hline Bajo peso & 2429 & $(3,7)$ \\
\hline Sobrepeso & 15195 & $(23,5)$ \\
\hline Obesidad & 5329 & $(8,2)$ \\
\hline Número de controles prenatales** & 7 & $(4,0)$ \\
\hline \multicolumn{3}{|l|}{ Control prenatal } \\
\hline $0-2$ controles & 8330 & $(12,8)$ \\
\hline $3-5$ controles & 12435 & $(19,2)$ \\
\hline $6-8$ controles & 20340 & $(31,4)$ \\
\hline 9 o más controles & 23565 & $(36,4)$ \\
\hline \multicolumn{3}{|l|}{ Enfermedad materna } \\
\hline Preeclampsia & 5740 & $(8,8)$ \\
\hline Eclampsia & 115 & $(0,2)$ \\
\hline Infección urinaria & 8549 & $(13,2)$ \\
\hline Diabetes gestacional & 235 & $(0,4)$ \\
\hline
\end{tabular}

*Media y desviación estándar (DE); ** Mediana y RIC y clínicas de las participantes en el estudio. La edad media de las gestantes fue de 25,7 años (DE $\pm 6,7)$. El grupo etario predominante fue de 20 a 35 años, lo cual representa el 69,3\%. En cuanto al grado de instrucción, un alto porcentaje cuenta con estudios secundarios completos (76,4\%). Además, un $64,5 \%$ de gestantes presentaba un IMC pregestacional normal, 23,5\% sobrepeso y un $8,2 \%$ obesidad. En cuanto al número de CPN se encontró una mediana de 7 con un rango intercuartílico de 4. Se observó que la mayor cantidad de participantes contaba con 9 a más controles $(36,4 \%)$, tan solo el $12,8 \%$ tuvieron controles entre 0 y 2 . Se encontraron 5740 casos de preeclampsia (8,8\%), así como 115 casos de eclampsia $(0,2 \%)$. Asimismo, un $13,2 \%$ de nuestras participantes padecieron infección urinaria durante su gestación.

Posteriormente se realizó el análisis bivariado en relación a los recién nacidos PEG (Tabla 2 ). Se encontró una asociación entre la edad materna $<20$ años y la condición de recién nacido PEG (RR 1,21; IC 95\%: 1,141,29). Asimismo, se encontró como factor de riesgo para PEG el ser divorciada, separada o soltera (RR 1,09; IC $95 \%: 1,03-1,16)$. Se encontró que tener un número de 0 a 2 CPN fue factor de riesgo para la condición de PEG al compararlo con un número de 6 a 8 CPN (RR 1,39; IC 95\%: 1,28-1,50). Asimismo, el tener 9 o más CPN actuó como factor protector (RR 0,73; IC 95\%: 0,68-0,79).

Tabla 2. Factores de riesgo a ser pequeño para la edad gestacional en gestantes atendidas en el Hospital María Auxiliadora: análisis crudo

\begin{tabular}{|c|c|c|c|c|c|c|c|}
\hline \multirow[b]{2}{*}{ Variables } & \multicolumn{2}{|c|}{ PEG } & \multicolumn{2}{|c|}{ AEG } & \multirow{2}{*}{\multicolumn{2}{|c|}{ RR (IC 95\%) }} & \multirow[b]{2}{*}{ Valor $p$} \\
\hline & $\mathbf{n}$ & $(\%)$ & $\mathbf{n}$ & $(\%)$ & & & \\
\hline \multicolumn{8}{|l|}{ Edad } \\
\hline 20-35 años & 3013 & $(7,9)$ & 35297 & $(92,1)$ & 1,00 & & \\
\hline Mayor de 35 años & 445 & $(8,3)$ & 4916 & $(91,7)$ & 1,05 & $(0,95-1,16)$ & 0,267 \\
\hline \multicolumn{8}{|l|}{ Estudios } \\
\hline Superior & 473 & $(7,7)$ & 5622 & $(92,3)$ & 1,00 & & \\
\hline Secundaria completa & 3556 & $(8,3)$ & 39256 & $(91,7)$ & 1,07 & $(0,97-1,17)$ & 0,148 \\
\hline Ninguno & 37 & $(9,5)$ & 353 & $(90,5)$ & 1,22 & $(0,88-1,68)$ & 0,216 \\
\hline \multicolumn{8}{|l|}{ Estado civil } \\
\hline Casada o conviviente & 2916 & $(8,0)$ & 33484 & $(92,0)$ & 1,00 & & \\
\hline Divorciada, separada o soltera & 1716 & $(8,8)$ & 17805 & $(91,2)$ & 1,09 & $(1,03-1,16)$ & 0,001 \\
\hline \multicolumn{8}{|l|}{ Controles prenatales } \\
\hline 6-8 controles & 1467 & $(8,3)$ & 16188 & $(91,7)$ & 1,00 & & \\
\hline $0-2$ controles & 858 & $(11,5)$ & 6588 & $(88,5)$ & 1,39 & $(1,28-1,50)$ & $<0,001$ \\
\hline 3-5 controles & 1101 & $(10,0)$ & 9914 & $(90,0)$ & 1,20 & $(1,12-1,30)$ & $<0,001$ \\
\hline 1-2 partos previos & 1494 & $(6,8)$ & 20626 & $(93,2)$ & 1,00 & & \\
\hline Nulípara & 2636 & $(9,5)$ & 25169 & $(90,5)$ & 1,40 & $(1,32-1,49)$ & $<0,001$ \\
\hline$\geq 3$ partos previos & 502 & $(8,4)$ & 5494 & $(91,6)$ & 1,23 & $(1,12-1,36)$ & $<0,001$ \\
\hline \multicolumn{8}{|l|}{ Índice de masa corporal } \\
\hline Normal & 3222 & $(8,7)$ & 33899 & $(91,3)$ & 1,00 & & \\
\hline Bajo peso & 287 & $(12,6)$ & 1996 & $(87,4)$ & 1,44 & $(1.29-1.62)$ & $<0,001$ \\
\hline Sobrepeso & 835 & $(6,7)$ & 11570 & $(93,3)$ & 0,77 & $(0,72-0,83)$ & $<0,001$ \\
\hline Obesidad & 288 & $(7,0)$ & 3824 & $(93,0)$ & 0,80 & $(0,71-0,90)$ & $<0,001$ \\
\hline \multicolumn{8}{|l|}{ Enfermedad materna } \\
\hline Preeclampsia & 787 & $(15,6)$ & 4256 & $(84,4)$ & 2,06 & $(1,92-2,21)$ & $<0,001$ \\
\hline Eclampsia & 30 & $(27,3)$ & 80 & $(72,7)$ & 3,30 & $(2,43-4,49)$ & $<0,001$ \\
\hline Infección urinaria & 609 & $(8,4)$ & 6681 & $(91,6$ & 1,00 & $(0,93-1,09)$ & 0,814 \\
\hline Diabetes gestacional & 9 & $(5,4)$ & 159 & $(94,6)$ & 0,64 & $(0,34-1,22)$ & 0,178 \\
\hline
\end{tabular}

PEG: pequeño para la edad gestacional; AEG: adecuado para edad gestacional 
Tabla 3. Factores de riesgo a ser pequeño para la edad gestacional en gestantes atendidas en el Hospital María Auxiliadora: análisis ajustado

\begin{tabular}{|c|c|c|c|}
\hline Variables* & $\mathbf{R R a}$ & (IC 95\%) & $\begin{array}{c}\text { Valor } \\
p\end{array}$ \\
\hline \multicolumn{4}{|l|}{ Edad } \\
\hline 20-35 años & 1,00 & & \\
\hline Menor de 20 años & 0,96 & $(0,90-1,04)$ & 0,384 \\
\hline Mayor de 35 años & 1,16 & $(1,04-1,29)$ & 0,004 \\
\hline \multicolumn{4}{|l|}{ Estado civil } \\
\hline Casada o conviviente & 1,00 & & \\
\hline Divorciada, separada o soltera & 1,03 & $(0,97-1,09)$ & 0,321 \\
\hline \multicolumn{4}{|l|}{ Control prenatales } \\
\hline 6-8 controles & 1,00 & & \\
\hline $0-2$ controles & 1,43 & $(1,32-1,55)$ & $<0,001$ \\
\hline 3-5 controles & 1,22 & $(1,14-1,32)$ & $<0,001$ \\
\hline$\geq 9$ controles & 0,74 & $(0,69-0,80)$ & $<0,001$ \\
\hline \multicolumn{4}{|l|}{ Paridad } \\
\hline 1 a 2 partos previos & 1,00 & & \\
\hline Nulípara & 1,32 & $(1,23-1,42)$ & $<0,001$ \\
\hline Tres o más partos previos & 1,12 & $(1,01-1,24)$ & 0,027 \\
\hline \multicolumn{4}{|l|}{ índice de masa corporal } \\
\hline Normopeso & 1,00 & & \\
\hline Bajo peso & 1,38 & $(1,23-1,54)$ & $<0,001$ \\
\hline Sobrepeso & 0,81 & $(0,75-0,88)$ & $<0,001$ \\
\hline Obesidad & 0,81 & $(0,72-0,92)$ & 0,001 \\
\hline \multicolumn{4}{|l|}{ Enfermedades maternas } \\
\hline Preeclampsia & 2,00 & $(1,86-2,15)$ & $<0,001$ \\
\hline Eclampsia & 3,22 & $(2,38-4,35)$ & $<0,001$ \\
\hline
\end{tabular}

*Variables que resultaron significativas en el análisis crudo

La paridad también mostró relación en cuanto a la incidencia de PEG, se encontró que las participantes nulíparas y las que tenías $\geq 3$ partos presentaron mayor riesgo ( $R R$ 1,40; IC 95\%: 1,32-1,49 y $R R$ 1,23; IC 95\%: 1,12-1,36 respectivamente). Asimismo, un IMC menor a 18,5 previo al embarazo presentó un RR de 1,44; IC 95\%: 1,29-1,62. En cuanto a las enfermedades maternas analizadas, se encontró que el haber presentado preeclampsia duplicó el riesgo para el recién nacido PEG (RR 2,06; IC 95\%: 1,92-2,21) y la eclampsia lo triplicó (RR 3,30; IC 95\%: 2,43-4,49). En cuanto a la infección urinaria y la diabetes gestacional no se encontró asociación con el recién nacido PEG.

Al ingresar las variables en el análisis ajustado (Tabla 3), se encontró que las participantes mayores de 35 años presentaron un mayor riesgo de PEG (RRa 1,16; IC 95\%: 1,04-1,29). Al analizar los CPN se observó que se mantuvo la asociación con los PEG. El tener de 0 a 2 CPN continuó siendo factor de riesgo al compararlo con 6 a 8 controles ( $\mathrm{RRa} 1,43$; IC 95\%: 1,32-1,55). Igualmente, el haber tenido solo de 3-5 CPN se mantuvo como factor de riesgo (RRa 1,22; IC 95\%: 1,14-1,32). Con 9 o más CPN el RRa fue 0,74 (IC 95\% 0,69-0,80) para PEG, con respecto a la categoría 6-8 CPN, permaneciendo como factor protector. En cuanto a la paridad se encontró que tanto las gestantes nulíparas (RRa 1,32; IC 95\%: 1,23-1,42) como las que tenían $\geq 3$ partos (RRa 1,12; IC 95\%: $1,01-1,24)$ presentaron mayor riesgo para PEG. Se encontró asociación entre el IMC previo y los PEG, siendo el bajo peso materno un factor de riesgo ( $R \mathrm{Ra}$ 1,38; IC 95\%: 1,23-1,54). Asimismo, el sobrepeso no se encontró asociado con un mayor riesgo para $\mathrm{PEG}$ (RRa 0,81; IC 95\%: 0,75-0,88). De las enfermedades estudiadas, la preeclampsia presentó un RRa de 2,0 (IC 95\% 1,86-2,15) y la eclampsia un RRa 3,22 (IC $95 \%: 2,38-4,35)$, siendo estos los factores de riesgo de mayor magnitud en este estudio.

\section{DISCUSIÓN}

De acuerdo con la curva de referencia peruana de peso al nacimiento para la edad gestacional en el presente estudio, se encontró una incidencia de $7,2 \%$ de recién nacidos a término PEG, similar a la hallada en otros estudios ${ }^{(2,7)}$. Dentro de los factores de riesgo para recién nacido a término PEG encontrados en esta población de gestantes, se halló a la preeclampsia y eclampsia. Estos factores de riesgo ya han sido evaluados en diversos estudios en los que señalan su asociación con PEG ${ }^{(9,12)}$. Se sostiene que el mecanismo fisiopatológico implicado es una disrupción de la vasculatura úteroplacentaria y/o un volumen sanguíneo materno reducido a nivel placentario ${ }^{(15)}$. Estos hallazgos, en relación a la alta incidencia de esta enfermedad en nuestro medio (16), refuerzan la importancia de mejorar las intervenciones diagnósticas y terapéuticas.

Otro resultado resaltante en el presente trabajo está en relación al número de $\mathrm{CPN}$. Diversos estudios señalan que las mujeres que reciben $\mathrm{CPN}$ tienen menores complicaciones perinatales ${ }^{(17-19)}$. En el Perú, el número de controles prenatales recomendado toma como referencia a la guía del Ministerio de Salud (MINSA), la cual recomienda seis controles, como mínimo ${ }^{(18)}$. En contraparte, el modelo de control prenatal propuesto por la OMS, enfocado a gestantes sin factores de riesgo, es de cuatro CPN ${ }^{(21,22)}$. Sin embargo, los datos sobre el número óptimo, frecuencia de visitas prenatales y contenido aún no están definidos. En el presente estudio se halló que tener de 0 a 2 CPN fue un factor de riesgo para $P E G$ al compararlo con un número de 6 a 8 CPN. Asimismo, con 3-5 CPN también se incrementó el riesgo, aunque en menor medida. Por otro lado, con 9 o más CPN el riesgo para PEG se redujo. Actualmente las investigaciones se encuentran enfocadas en determinar el número adecuado de CPN y los tipos de intervenciones más adecuadas $(17,19,21,22)$. Este estudio encontró que a medida que la categoría tiene menor número de $\mathrm{CPN}$ el riesgo para $\mathrm{PEG}$ fue mayor. En vista que un número reducido de $\mathrm{CPN}$ es un factor de riesgo 
modificable, es importante enfocar las políticas de salud a la atención prenatal.

Otro hallazgo dentro del estudio fue la relación entre la nuliparidad y el recién nacido PEG. Encontramos un riesgo mayor en gestantes nulíparas, lo cual coinciden con otros estudios $(9,11,23)$. Las razones fisiológicas que podrían explicar esta relación están dadas probablemente por un volumen uterino menor con respecto a mujeres con paridad previa. Acorde con este concepto, otros estudios encontraron que un número de 3 o más partos fue un factor protector para la condición de $\mathrm{PEG}^{23}$. Paradójicamente, en nuestro análisis, las gestantes que tuvieron tres o más partos presentaron un riesgo mayor para tener un recién nacido PEG. Se requieren más estudios en nuestro medio que amplíen el análisis entre paridad y la condición de PEG.

En el análisis, se encontró una asociación entre un bajo peso pregestacional y un recién nacido a término PEG. A nivel regional, se describe el hallazgo de un bajo peso materno asociado al recién nacido con bajo peso, pero no se estudia una asociación directa con el recién nacido PEG ${ }^{(24,25)}$. Por otro lado, en poblaciones de Norteamérica, Europa y Asia se reportan estudios que indican que un peso pregestacional bajo es un factor de riesgo para tener un recién nacido PEG ${ }^{(13,14,26,27)}$. Además, en este estudio se encontró que tanto el sobrepeso como la obesidad no fueron factores de riesgo para PEG. En otros estudios, estos mismos factores fueron identificados como factores de riesgo para el recién nacido GEG ${ }^{(26,27)}$. Una limitación del estudio fue no evaluar la ganancia de peso materno durante la gestación, ya que requería un mínimo de dos controles, uno al inicio y otro al final de la gestación; para lo cual se hubiera excluido un número significativo de participantes del estudio, dando lugar a un posible sesgo de selección.

Con respecto a factores sociodemográficos, este estudio encontró que una edad materna mayor a 35 años es un factor de riesgo para tener un recién nacido PEG. A pesar de que existen estudios en nuestra región que reporten asociación entre las gestantes mayores de 35 años y diversas complicaciones perinatales, son escasos los que buscan una asociación específica con los PEG ${ }^{(28,29)}$. Los hallazgos de este estudio podrían estar asociados con una disminución en el potencial para el crecimiento fetal, lo que posiblemente refleja un envejecimiento biológico de los tejidos y sistemas maternos.

En la población estudiada se halló una incidencia de diabetes gestacional de $0,4 \%$, la cual difiere de los hallazgos de otros estudios. Este resultado representa una limitación, puesto que la incidencia encontrada en la región es mucho mayor. Un estudio local reportó 4,8\% de diabetes gestacional ${ }^{(30)}$, mientras que en Argentina se reportó $5,9 \%{ }^{(24)}$. En poblaciones norteamericanas se reporta una prevalencia de 6 a $7 \%{ }^{(29)}$. Esta gran diferencia se sustenta, posiblemente, en la ausencia de un screening y estudios diagnósticos adecuados. A pesar de ello, en la mayoría de estudios esta variable se ha visto relacionada con complicaciones como el GEG, macrosomía, anomalías fetales, entre otros; mas no con el PEG, por lo que no debió afectar los resultados del presente estudio. De manera similar, la incidencia de restricción del crecimiento intrauterino en el estudio fue casi nula, probablemente por deficiencias en los criterios diagnósticos del hospital. Por esto, al corroborar su irrelevancia en los resultados finales, no se utilizó en el estudio.

Otra limitación importante es que los participantes del estudio corresponden a un hospital de referencia, por lo cual se esperaría una mayor comorbilidad. Sin embargo, al comparar las características de la población, así como la incidencia de enfermedades observamos que no difieren con las de otros estudios locales ${ }^{(32,33)}$. Por otro lado, si bien el tamaño muestral de este estudio fue importante y se encontraron diversos factores de riesgo relacionados al recién nacido $P E G$, algunas variables podrían ser mejor evaluadas mediante una cohorte prospectiva, con el fin de mejorar el nivel de evidencia.

Asimismo, posterior al diseño y análisis del presente trabajo, un estudio reciente de la OMS publicó nuevas curvas antropométricas y nuevos estándares de referencia para el diagnóstico del PEG. Sería recomendable establecer futuros estudios que permitan una evaluación comparativa de estas nuevas curvas con las de Ticona y Lubchenco ${ }^{(2,6,34)}$.

En conclusión, en este estudio los factores de riesgo más importantes fueron la preeclampsia y eclampsia. Otros factores identificados fueron la edad materna $\geq 35$ años, el bajo peso pregestacional, un número reducido de CPN, la nuliparidad y el antecedente de $\geq 3$ partos previos. Estos hallazgos fueron concordantes con diversos estudios a nivel mundial. Los factores de riesgo para el recién nacido PEG identificados en este estudio constituyen una base para el desarrollo de estrategias de intervención pertinentes, con la finalidad de disminuir la condición dePEG. Se debe identificar a las gestantes con mayor riesgo y actuar rigurosamente, poniendo especial énfasis en factores modificables, tales como la frecuencia de sus controles prenatales.

Contribuciones de autoría: JETM, OPM y MAD tuvieron la idea del estudio y obtuvieron la data, PMT realizó el análisis e interpretación de datos. Todos los autores participaron en la redacción, aportes críticos y aprobación de la versión final a publicar.

Fuente de financiamiento: autofinanciado

Conflictos de interés: los autores declaran no tener conflictos de interés con la publicación de este artículo. 


\section{REFERENCIAS BIBLIOGRÁFICAS}

1. Martin JA, Hamilton BE, Sutton PD, Ventura SJ, Mathews TJ, Osterman MJ. Births: final data for 2008. Natl Vital Stat Rep. 2010 Dec 8;59(1):1, 3-71.

2. Ticona-Rendón M, Huanco-Apaza D. Curva de referencia peruana del peso de nacimiento para la edad gestacional y su aplicación para la identificación de una nueva población neonatal de alto riesgo. Rev Peru Med Exp Salud Pública. 2007;24(4):325-35.

3. Mathews TJ, MacDorman MF. Infant mortality statistics from the 2005 period linked birth/infant death data set. Natl Vital Stat Rep. 2008;57(2):1-32.

4. Tsai LY, Chen YL, Tsou KI, Mu SC; for the Taiwan Premature Infant Developmental Collaborative Study Group. The Impact of Small-forgestational-age on Neonatal Outcome Among Very-low-birth-weight Infants. Pediatrics \& Neonatology. 2014;56(2):101-7.

5. Williams RL, Creasy RK, Cunningham GC, Hawes WE, Norris FD, Tashiro M. Fetal growth and perinatal viability in California. Obstet Gynecol.1982;59(5):624-32.

6. Battaglia FC, Lubchenco LO. A practical classification of newborn infants by weight and gestational age. J Pediatr. 1967;71(2):159-63.

7. Boguszewski MCS, Merico V, Bergada I, Damiani D, Belgorosky A, González $\mathrm{P}$, et al. Consenso Latinoamericano: niños pequeños para la edad gestacional. Rev Chil Pediatr. 2012;83(6):620-34.

8. Heaman M, Kingston D, Chalmers B, Sauve R, Lee L, Young D. Risk factors for preterm birth and small for gestational age births among Canadian women. Paediatr Perinat Epidemiol. 2013;27(1):54-61. doi: 10.1111/ ppe.12016.

9. Ota E, Ganchimeg T, Morisaki N, Vogel JP, Pileggi C, Ortiz-Panozo E, et al. Risk factors and adverse perinatal outcomes among term and preterm infants born small-for-gestationalage: secondary analyses of the WHO Multi-Country Survey on Maternal and Newborn Health. PLoS One. 2014;9(8):e105155. doi: 10.1371/ journal.pone.0105155.

10. Hung TH, Hsieh TT, Lo LM, Chiu TH, Hsieh CC, Hsu JJ. Risk factors and perinatal outcomes associated with idiopathic small for gestational age Taiwanese newborns. Int J Gynaecol Obstet. 2013;122(3):212-5. doi: 10.1016/j.ijgo.2013.03.033.

11. Isaksen CV, Laurini RN, Jacobsen G. Pre-pregnancy risk factors of smallfor-gestational-age births and perinatal mortality. Acta Obstet Gynecol Scand Suppl. 1997;165:44-9.

12. Thompson JM, Clark PM, Robinson E, Becroft DM, Pattison NS, Glavish N, et al. Risk factors for small-for-gestational-age babies: The Auckland Birthweight Collaborative Study. J Paediatr Child Health. 2001;37(4):369-75.

13. Abrams B, Newman V. Small-forgestational-age birth: maternal predictors and comparison with risk factors of spontaneous preterm delivery in the same cohort. Am J Obstet Gynecol. 1991;164(3):78590.

14. Fescina RH, De Mucio B, Martínez G, Díaz JL, Mainero L, Rubino M. Sistema informático perinatal (SIP): historia clínica perinatal: instrucciones de llenado y definición de términos. Montevideo: CLAP/SMR; 2010.

15. Von Dadelszen P, Ornstein MP, Bull SB, Logan AG, Koren G, Magee LA. Fall in mean arterial pressure and fetal growth restriction in pregnancy hypertension: a meta-analysis. Lancet. 2000;355(9198):87-92.

16. Pacheco J, Wagner P, Williams N, Sánchez S. Enfermedades hipertensivas de la gestación. En: Pacheco J, editor. Ginecología y Obstetricia. 2da ed. Lima: MAD Corp SA; 2006. 17. Bergsjo P. What is the evidence for the role of antenatal care strategies in the reduction of maternal mortality and morbidity? Norway: John Snow, Inc.

18. Sánchez-Nuncio HR, Pérez-Toga G, Pérez-Rodriguez P, Vázquez-Nava F. [Impact of the prenatal care in the neonatal morbidity and mortality]. Rev Med Inst Mex Seguro Soc. 2005;43(5):377-80. [Artículo en español]

19. Dowswell T, Carroli G, Duley L, Gates S, Gülmezoglu AM, Khan-Neelofur $\mathrm{D}$, et al. Alternative versus standard packages of antenatal care for low-risk pregnancy. Cochrane Database Syst
Rev. 2010; 6(10):CD000934. doi: 10.1002/14651858.CD000934.pub2.

20. Perú, Ministerio de Salud. Guías Nacionales de Atención Integral de la Salud Sexual y Reproductiva. Lima: MINSA; 2004.

21. Villar J, Carroli G, Khan-Neelofur D, Piaggio G, Gülmezoglu M. Patterns of routine antenatal care for low-risk pregnancy. Cochrane Database Syst Rev. 2001;(4):CD000934.

22. Villar J, Ba'aqeel H, Piaggio G, Lumbiganon P, Miguel Belizán J, Farnot U, et al. WHO antenatal care randomised trial for the evaluation of a new model of routine antenatal care. Lancet. 2001;357(9268):1551-64.

23. Kozuki N, Lee AC, Silveira MF, Sania A, Vogel JP, Adair L, et al. The associations of parity and maternal age with small-for-gestational-age, preterm, and neonatal and infant mortality: a meta-analysis. BMC Public Health. 2013;13(Suppl 3):S2.

24. Sánchez Jaeger A, Del Real Vargas SI, Solano Rodríguez L, Peña Perdomo E, Barón MA. Índice de Masa Corporal al comienzo del embarazo en un grupo de gestantes venezolanas de bajo estrato socioeconómico y su relación con la antropometría de sus recién nacidos. Arch Latinoam Nutr. 2006;56(2):141-5.

25. Grandi CA. Relación entre la antropometría materna y la ganancia de peso gestacional con el peso de nacimiento, y riesgos de peso bajo al nacer, pequeño para la edad gestacional y prematurez en una población urbana de Buenos Aires. Arch Latinoam Nutr. 2003;53(4):369-75.

26. Park JH, Lee BE, Park HS, Ha EH, Lee SW, Kim YJ. Association between pre-pregnancy body mass index and socioeconomic status and impact on pregnancy outcomes in Korea. J Obstet Gynaecol Res. 2011;37(2):138-45. doi: 10.1111/j.1447-0756.2010.01332.x.

27. Yu Z, Han S, Zhu J, Sun X, Ji C, Guo $\mathrm{X}$. Pre-pregnancy body mass index in relation to infant birth weight and offspring overweight/obesity: a systematic review and meta-analysis. PLoS One. 2013;8(4):e61627. doi: 10.1371/journal.pone.0061627.

28. Chamy V, Cardemil F, Betancour P, Ríos M, Leighton L. Riesgo obstétrico y perinatal en embarazadas mayores 
de 35 años. Rev Chil Obstet Ginecol. 2009;74(6):331-8.

29. Jacobsson B, Ladfors L, Milsom I. Advanced maternal age and adverse perinatal outcome. Obstet Gynecol. 2004;104(4):727-33.

30. Ylave G, Bertha R. Diabetes mellitus gestacional, experiencia en el Hospital Militar Central. Rev Per Ginecol Obstet. 2009;55:135-42.

31. Moyer VA; U.S. Preventive Services Task Force. Screening for gestational diabetes mellitus: U.S. Preventive Services Task Force recommendation statement. Ann Intern Med.
2014;160(6):414-20. doi: 10.7326/ M13-2905.

32. Pacheco J. Preeclampsia/eclampsia: Reto para el ginecoobstetra. Acta Med Per. 2006;23(2):100-11.

33. Echevarria-Zarate J, Sarmiento Aguilar E, Osores-Plengue F. Infección del tracto urinario y manejo antibiótico. Acta Med Per. 2006; 23(1):26-31.

34. Villar J, Cheikh Ismail L, Victora CG, Ohuma EO, Bertino E, Altman DG, et al. International standards for newborn weight, length, and head circumference by gestational age and sex: the
Newborn Cross-Sectional Study of the INTERGROWTH-21st Project. Lancet. 2014;384(9946):857-68. doi: $10.1016 /$ S0140-6736(14)60932-6.

Correspondencia: José Eduardo TejedaMariaca

Dirección: Jr. Las Petunias 200, Urb. El Palmar, Santiago de Surco, Lima, Perú Teléfono: (51) 987332142

Correo electrónico: tejedaeduardo88@ hotmail.com

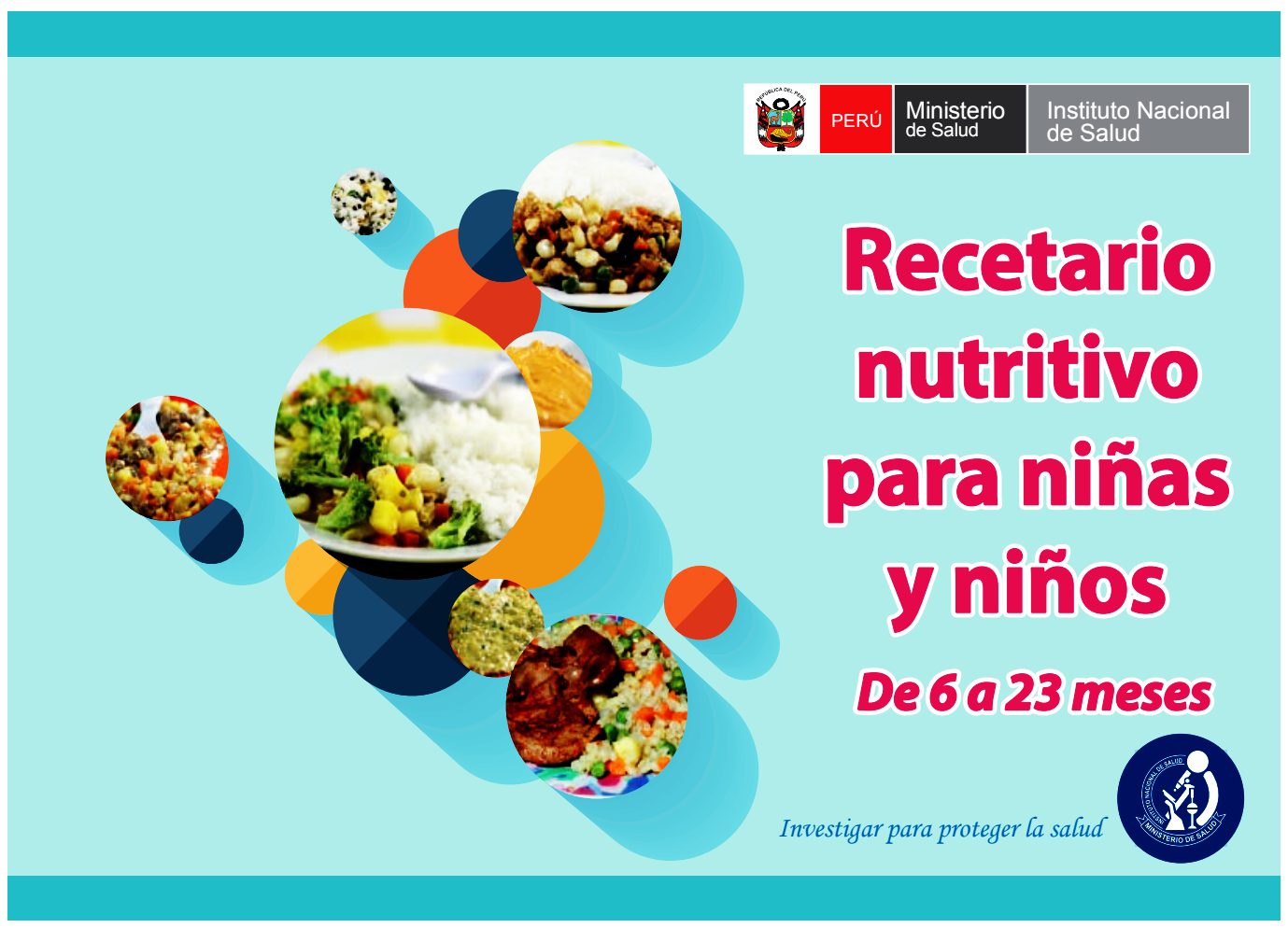

